\title{
Triglycerides constituted of short and medium chain fatty acids and dicarboxylic acids in Momordica charantia, as well as capric acid, inhibit $\mathrm{PGE}_{2}$ production in RAW264.7 macrophages
}

\author{
Wen-Huey $\mathrm{Wu}^{\mathrm{a}}$, Bi-Yu Lin ${ }^{\mathrm{a}}$, Yueh-Hsiung Kuo ${ }^{\text {b,c,1 }}$, Ching-jang Huang ${ }^{\mathrm{d}, *, 1}$ \\ ${ }^{a}$ Graduate Program of Nutrition, Department of Human Development and Family Studies, National Taiwan Normal University, 162, Sec. 1, Hoping E. Rd., Taipei 106, Taiwan \\ ${ }^{\mathrm{b}}$ Tsuzuki Institute for Traditional Medicine, College of Pharmacy, China Medical University, 91, Hsueh-Shih Rd., Taichung 404, Taiwan \\ ${ }^{\mathrm{c}}$ Agricultural Biotechnology Research Center, Academia Sinica, Taipei 115, Taiwan \\ ${ }^{\mathrm{d}}$ Department of Biochemical Science and Technology and Institute of Microbiology and Biochemistry, National Taiwan University, 1, Sec. 4, Roosevelt Rd., Taipei 106, Taiwan
}

\section{A R T I C L E I N F O}

\section{Article history:}

Received 2 November 2008

Received in revised form 1 March 2009

Accepted 1 April 2009

Available online $\mathrm{xxxx}$

\section{Keywords:}

Momordica charantia

Bitter gourd

Macrophages

Prostaglandin $\mathrm{E}_{2}$

Capric acid

\begin{abstract}
A B S T R A C T
This study was aimed to identify compounds in bitter gourd (Momordica charantia) ethyl acetate extract (EAE), which inhibit lipopolysaccharide-induced prostaglandin $\mathrm{E}_{2}\left(\mathrm{PGE}_{2}\right)$ production in RAW264.7 cells. Bitter gourd EAE was partitioned between $n$-hexane and methanol $/ \mathrm{H}_{2} \mathrm{O}(90 / 10)$. The hexane fraction was further separated by repeated silica gel chromatographies, and a reverse phase (RP) C18 chromatography. Fraction RP-10 showed the highest inhibition effect on $\mathrm{PGE}_{2}$ production (Max inhibition $=96 \%$, $\mathrm{IC}_{50}=2.3 \mu \mathrm{g} / \mathrm{ml}$ ) and was identified to be triglycerides constituted of short and medium chain fatty acids by ${ }^{1} \mathrm{H}$ NMR, IR and H-HCOSY, and dicarboxylic acids by GC/MS. Fatty acids with 3-20 carbons were tested for the inhibitory activity, and capric acid exhibited the highest effect (Max inhibition $=99 \%$, $\mathrm{IC}_{50}=6.5 \mu \mathrm{M}$ ). In conclusion, triglycerides composed of short and medium chain fatty acids and dicarboxylic acids in bitter gourd inhibit $\mathrm{PGE}_{2}$ production, and capric acid is the most potent inhibitor among the fatty acids.
\end{abstract}

(c) 2009 Elsevier Ltd. All rights reserved.

\section{Introduction}

Prostaglandin $\mathrm{E}_{2}\left(\mathrm{PGE}_{2}\right)$ is an important pro-inflammatory mediator in many chronic inflammatory conditions, including rheumatoid arthritis (Robinson, McGuire, \& Levine, 1975), inflammatory bowel diseases (Subbaramaiah et al., 2004), cancer (Sinha, Clements, Fulton, \& Ostrand-Rosenberg, 2007), atherosclerosis (Gómez-Hernández et al., 2006), atherosclerotic plague rupture (Cipollone et al., 2004) and age-related degeneration (Wu \& Meydani, 2004). In response to intrinsic cytokines (Arias-Negrete, Keller, \& Chadee, 1995), or extrinsic factors, such as lipopolysaccharide (LPS) (Rhee \& Hwang, 2000), macrophages release large amounts of $\mathrm{PGE}_{2}$. $\mathrm{PGE}_{2}$ also stimulates the production of proinflammatory cytokines (Williams \& Shacter, 1997). Inhibition of the production of $\mathrm{PGE}_{2}$ has been used to ameliorate inflammatory symptoms and suppress related diseases (Guadagni et al., 2007).

Bitter gourd (Momordica charantia) is a very common oriental vegetable in tropical and subtropical areas. It is traditionally regarded as a "cooling" or "fire-reducing" food. In our previous study, that characterised "heating" and "cooling" foods by their effects on

\footnotetext{
* Corresponding author. Tel.: +88622362 1301.

E-mail address: cjjhuang@ntu.edu.tw (C.-j. Huang).

Equal contribution to this article.
}

$\mathrm{PGE}_{2}$ production in LPS-stimulated RAW264.7 macrophages, we found that ethyl acetate extract (EAE) of bitter gourd inhibited $\mathrm{PGE}_{2}$ production (Huang \& $\mathrm{Wu}, 2002$ ). This study was thus aimed to further investigate the active components in bitter gourd EAE that inhibit $\mathrm{PGE}_{2}$ production.

\section{Materials and methods}

\subsection{Materials}

Wild bitter gourds ( $M$. charantia L., strain Hualian No. 4), were kindly provided by Mr. Chong-Ho Chuan of Hualien District Agricultural Research \& Extension Station. ${ }^{1} \mathrm{H}$ NMR and H-HCOSY spectra were obtained on Bruker AM-300 and Bruker-500 instruments, respectively. An HP 6890 series gas chromatograph (GC) equipped with a $30 \mathrm{~m} \times 0.25 \mathrm{~mm} \times 0.25 \mu \mathrm{m}$ HP- 5 MS column was used for the analysis of fatty acids. Mass spectral analysis was done on a JEOL JMS-HX300 mass spectrometer. Thin-layer chromatography (TLC) was performed on silica gel 60F254 TLC plates (Merck, Darmstadt, Germany). Silica gel $(\sim 230-400 \mu \mathrm{m})$ (Macherey-Nagel, Germany) and reverse phase C18 were used for column chromatography. Infrared spectra were obtained on a Bio-Rad FTS-40 FTIR. The RAW 264.7 macrophage cell line (CCRC60001, originally from the American Type Culture Collection; designation, TIB-71) 
was obtained from the cell bank of the Food Industry Research and Development Institute, Hsin Chu, Taiwan. Dulbecco's minimal essential medium (DMEM) and fetal bovine serum (FBS) were purchased from Gibco (Gaithersburg, MD, USA). LPS (Escherichia coli, serotype 026: B6) was purchased from Sigma-Aldrich (St. Louis, MO, USA). Fatty acids, propionic acid (3:0), butyric acid (4:0), caproic acid (6:0), caprylic acid (8:0), capric acid (10:0), lauric acid (12:0), myristic acid (14:0), palmitic acid (16:0) and dicarboxylic acids, octanedioic acid (suberic acid), nonanedioic acid (azelainic acid) and decanedioic acid (sebacic acid), were purchased from Sigma-Aldrich; oleic acid (18:1), linoleic acid (18:2n6), arachidonic acid (20:4n6) and conjugated linoleic acid (CLA) were purchased from Cayman (Ann Arbor, MI, USA); medium chain triglycerides (MCT) containing 67\% caprylic acid (8:0), and 23\% capric acid (10:0) were purchased from Mead Johnson (Evansville, IL, USA). Conjugated linolenic acid (CLN) was isolated and purified from bitter gourd in our laboratory, previously (Chuang et al., 2006); it was composed of $77 \%$ 9c, $11 \mathrm{t}, 13 \mathrm{t}-\mathrm{CLN}, 11 \%$ stearic acid and $12 \%$ palmitic acid, based on the mass spectral data.

\subsection{Preparation of water-soluble and ethyl acetate-soluble fractions of bitter gourd}

Fresh whole bitter guard was homogenised and filtered through cotton cloth. The juice was centrifuged and the supernatant was lyophilised to obtain the water extract (WE). Lyophilised whole bitter guard was extracted with ethyl acetate (EA) for $48 \mathrm{~h}$. The extract was filtered through Whatman no. 1 filter paper, followed by removing solvent in a rotary vacuum evaporator (Buchi, Essen, Germany), and ethyl acetate extract (EAE) was obtained. WE and EAE were tested for their effects on LPS-stimulated $\mathrm{PGE}_{2}$ production in RAW264.7 cells.

\subsection{Fractionation of bitter gourd ethyl acetate extract (EAE) in large quantity}

One hundred and thirteen kilograms of bitter gourd were lyophilised to produce $7.8 \mathrm{~kg}$ of powder. The powder was extracted with EA twice $(2 \times 801)$ at room temperature for two days. The pooled extracts were evaporated in a rotary evaporator to remove the solvent and yielded $250 \mathrm{~g}$ of EAE. EAE was suspended in 11 of $n$-hexane, and partitioned with 11 of methanol $(\mathrm{MeOH}) / \mathrm{H}_{2} \mathrm{O}(90 / 10)$ three times. The resulting two fractions were evaporated to yield $234 \mathrm{~g}$ of $n$-hexane extract and $6 \mathrm{~g}$ of $\mathrm{MeOH} / \mathrm{H}_{2} \mathrm{O}(90 / 10)$ extract. Hexane extract was subsequently chromatographed over silica gel with an EA/hexane gradient solvent system and finally an EA/ $\mathrm{MeOH}(90 / 10)$ solvent. The eluted fractions showing similar thinlayer chromatography (TLC) and ${ }^{1} \mathrm{H}$ NMR patterns were collected, evaporated and weighed; the evaporated crude compounds were screened for their inhibition effects upon LPS-stimulated $\mathrm{PGE}_{2}$ production in the RAW264.7 macrophages.

Active fractions F189-191 (8.3 g) eluted by EA/MeOH (90/10) were combined, dissolved in acetone, subjected to a second silica gel column chromatography, eluted first by chloroform/ethanol (6/1), to yield two subfractions, F1-6 (4.3 g) and F8-12 (0.4 g), and then eluted by chloroform/ethanol (5/1) to yield F13-21 (2.8 g). F1-6 and F8-12, but not F13-21, were found to have the $\mathrm{PGE}_{2}$ inhibition effect in macrophages. F1-6 (1.7 g) were dissolved in ethyl acetate, subjected to a third silica gel column chromatography, and eluted by EA/hexane (60/40). Fractions with similar TLC patterns were combined to yield seven fractions, and tested for the $\mathrm{PGE}_{2}$ inhibition effect in macrophages. The three active fractions F1-6-2 (247 mg), F1-6-3 (237 mg) and F1-6-4 (258 mg) were combined $(742 \mathrm{mg}$ ) and subjected to a fourth silica gel column chromatography, successively eluted by an EA/hexane gradient, starting at $90 \%$ hexane with a 5\% increase in EA for every 12 fractions ( $150 \mathrm{ml} /$ fraction) collected. Fractions with similar TLC patterns were combined to yield 16 fractions. The active fractions p11-13 (220 mg) were combined and subjected to a reverse phase (RP) $\mathrm{C} 18$ chromatography, eluted by $\mathrm{MeOH} / \mathrm{H}_{2} \mathrm{O}(80 / 20), \mathrm{MeOH} /$ $\mathrm{H}_{2} \mathrm{O}(90 / 10)$ and $100 \% \mathrm{MeOH}$. Fraction RP-10 (19.7 mg), eluted by $100 \% \mathrm{MeOH}$, showed the highest $\mathrm{PGE}_{2}$ inhibition effect. RP-10 was analysed by ${ }^{1} \mathrm{H}$ NMR and H-HCOSY for structural identification. Fraction F1-6 was subjected to GC/MS for fatty acid analysis after saponification and methylation by the diazomethane method (Mueller, 1996).

\subsection{Cell culture}

RAW 264.7 cells were seeded on 96 well plates at a concentration of $5 \times 10^{5}$ cells $/ \mathrm{ml}$, and incubated for $24 \mathrm{~h}$ at $37^{\circ} \mathrm{C}$ in $5 \% \mathrm{CO}_{2}$. The unattached cells were washed away and the cells were treated with serum-free Dulbecco's modified Eagle's medium (DMEM) containing bitter guard extracts or fatty acids in the present or absence of $100 \mathrm{ng} / \mathrm{ml}$ of LPS for $18 \mathrm{~h}$. Then, the medium was collected and analysed for $\mathrm{PGE}_{2}$ using a commercial enzyme immunoassay kit (Cayman, Ann Arbor, Mich., USA). Cell viability and cell numbers after treatments were assayed using the MTT method (Denizot \& Lang, 1986).

\subsection{Statistical methods}

Data were expressed as means \pm SD. Experiments were repeated at least three times in triplicate. The significance of difference was analysed by one way analysis of variance (ANOVA) and a Duncan's multiple rank test, using SPSS software. To calculate the percentage inhibition of LPS-stimulated $\mathrm{PGE}_{2}$ production, $\mathrm{PGE}_{2}$ production in the presence of $100 \mathrm{ng} / \mathrm{ml}$ of LPS was taken as $0 \%$ inhibition and basal $\mathrm{PGE}_{2}$ production as $100 \%$ inhibition; the percentage inhibition by a sample at a specific concentration was calculated as [1 - (PGE ${ }_{2}$ sample with LPS-PGE 2 basal)/(PGE 2 LPS only - PGE $_{2}$ basal) $] \times 100 \%$. The $\mathrm{IC}_{50}$ value, calculated from the curve of percentage inhibition versus concentration, is the concentration of sample that results in 50\% inhibition.

\section{Results}

\subsection{Culture conditions for measuring $P G E_{2}$}

$\mathrm{PGE}_{2}$ production induced by LPS $(100 \mathrm{ng} / \mathrm{ml})$ in RAW264.7 was tested for $0,12,18$, and $24 \mathrm{~h}$ and the maximal production of $\mathrm{PGE}_{2}$

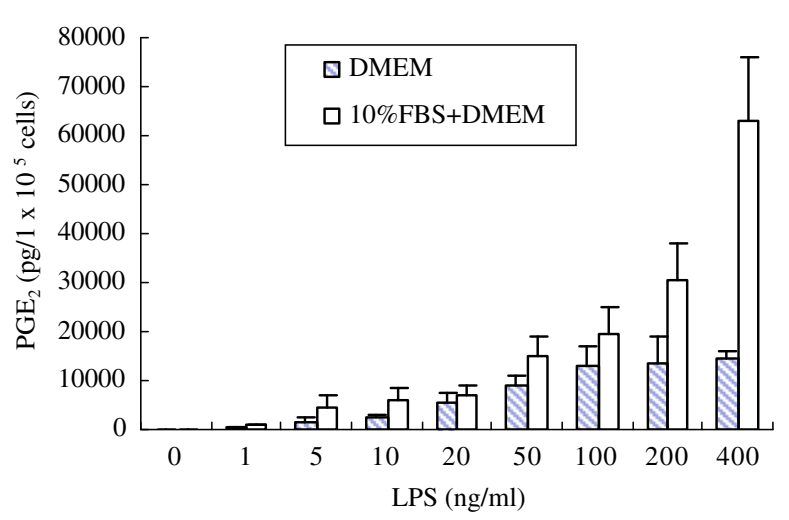

Fig. 1. $\mathrm{PGE}_{2}$ production induced by various concentrations of LPS in RAW264.7 cells cultured with or without the addition of fetal bovine serum (FBS). The cells were treated with LPS at $0-400 \mathrm{ng} / \mathrm{ml}$ with or without $10 \%$ FBS for $18 \mathrm{~h}$ and medium was collected for $\mathrm{PGE}_{2}$ analysis using an EIA. At least three batches of separate experiments were carried out with similar results. The values are means \pm SD of triplicates in a representative experiment. 

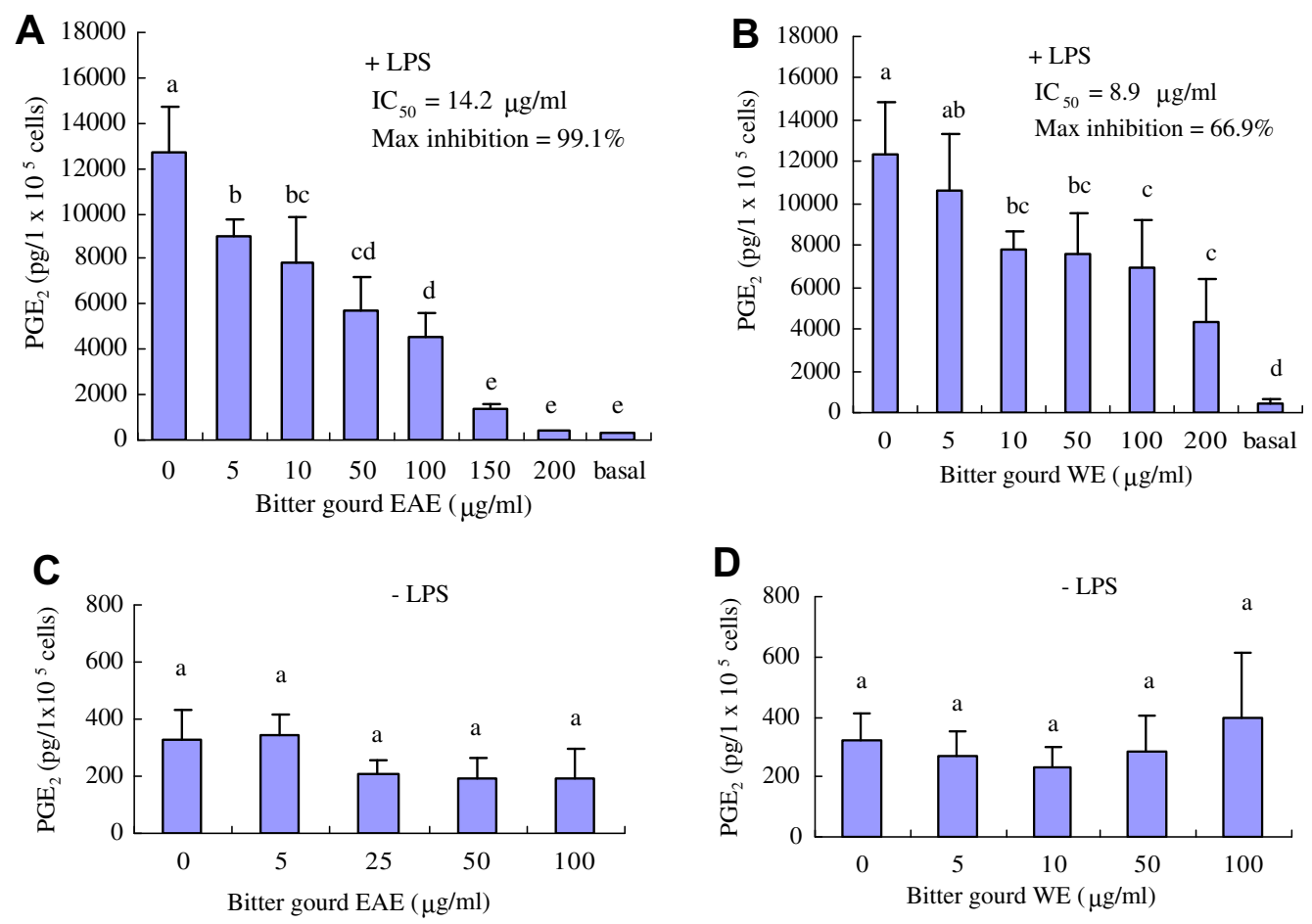

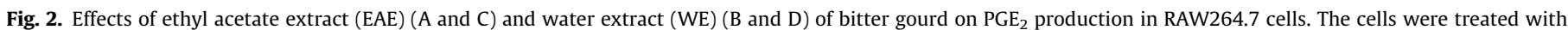

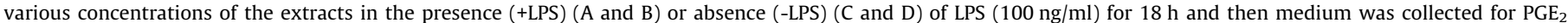

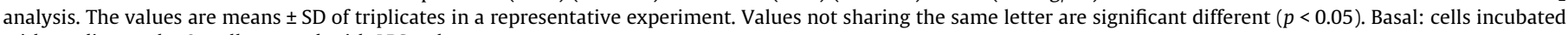
with medium only. 0: cells treated with LPS only.

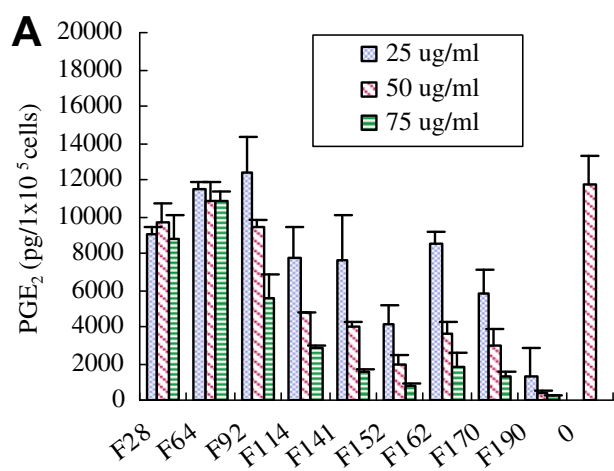

Fractions from hexane extracts

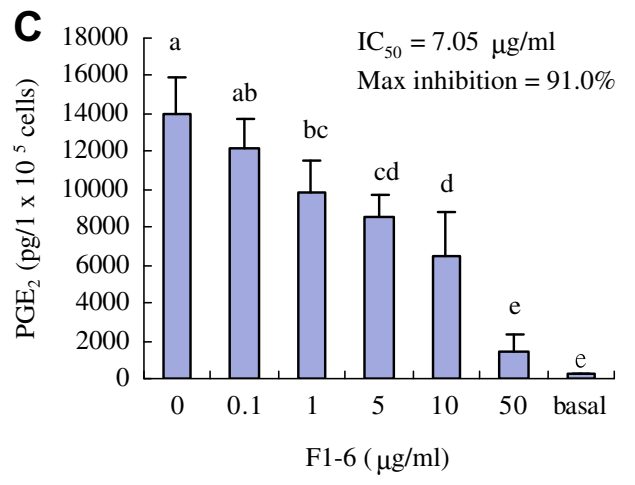

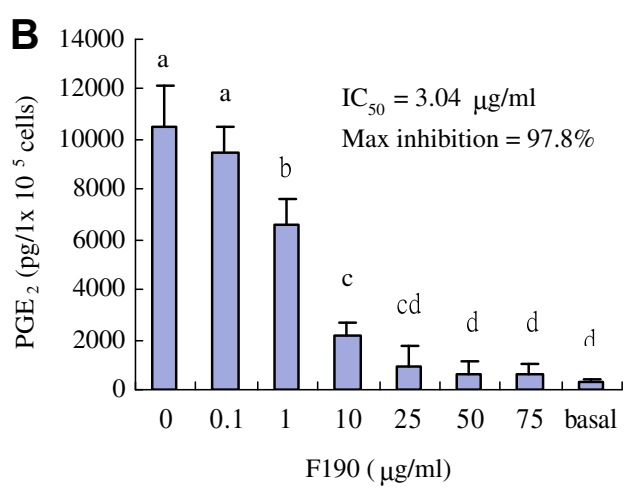

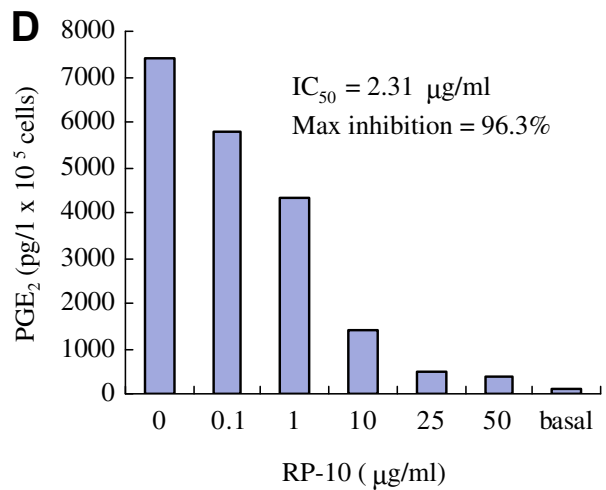

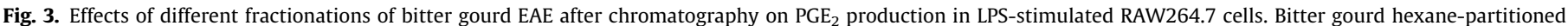

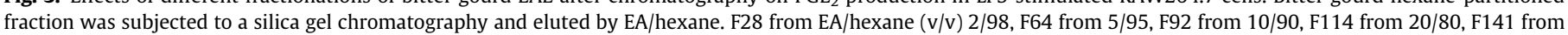

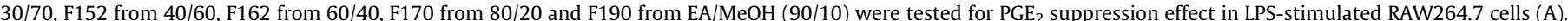

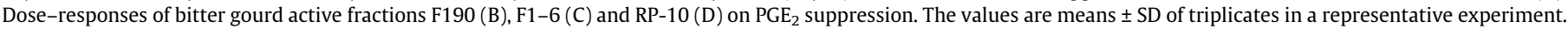
Values not sharing the same letter are significant different $(p<0.05)$. Basal: cells incubated with medium only. 0 : cells treated with $100 \mathrm{ng} / \mathrm{ml}$ LPS only. 
was found to occur at $18 \mathrm{~h}$ (data not shown). $\mathrm{PGE}_{2}$ production induced by LPS at concentrations of $0,1,5,10,20,50,100,200$, $400 \mathrm{ng} / \mathrm{ml}$ in RAW264.7, incubated with or without 10\% FBS for $18 \mathrm{~h}$, was tested. At LPS concentration of $100 \mathrm{ng} / \mathrm{ml}$, the production of $\mathrm{PGE}_{2}$ in cells cultured without FBS reached peak level (Fig. 1) but, in cells cultured with FBS, $\mathrm{PGE}_{2}$ production increased dramatically as the concentration of LPS increased. To avoid over stimulation, culture medium without FBS was used during LPS stimulation in the following experiments. The concentrations of extracts used to culture cells were those which did not cause cytotoxicity examined by the MTT assay.

\subsection{Inhibition of $P G E_{2}$ production by bitter gourd extracts and the separated fractions}

Both kinds of bitter gourd extracts, WE and EAE, decreased the secretion of $\mathrm{PGE}_{2}$ in LPS-stimulated RAW264.7 (Fig. 2A and B), but the EAE had higher inhibition activity than had WE. Without LPS stimulation, the basal $\mathrm{PGE}_{2}$ levels were low and not affected by either extract (Fig. 2C and D).

After partition of EAE, the hexane-soluble fraction showed higher inhibitory effect than did the $\mathrm{MeOH} / \mathrm{H}_{2} \mathrm{O}$ (90/10)-soluble fraction (data not shown). The hexane-soluble fraction was then separated by a silica gel chromatography. The fraction F190, eluted by $\mathrm{EA} / \mathrm{MeOH}(90 / 10)$, showed the highest $\mathrm{PGE}_{2}$ inhibitory activity (Fig. 3A), with maximal inhibition of $97.8 \%$ and $\mathrm{IC}_{50}$ of $3.04 \mu \mathrm{g} / \mathrm{ml}$ (Fig. 3B). Then, fractions F189-191, with similar patterns on TLC, were pooled and separated by a second silica gel chromatography. The obtained fraction F1-6, eluted by chloroform/ethanol (6/1), had the highest activity (maximal inhibition $=91.0 \%$ and $\mathrm{IC}_{50}=7.05 \mu \mathrm{g} / \mathrm{ml}$ ) (Fig. 3C). The fraction F1-6 was further separated by two successive silica gel chromatographies and finally a reverse phase chromatography. The fraction RP-10, eluted with methanol, showed the highest $\mathrm{PGE}_{2}$ inhibition effect (maximal inhibition $=96.3 \%$ and $\mathrm{IC}_{50}=2.31 \mu \mathrm{g} / \mathrm{ml}$ ) (Fig. 3D).

\subsection{Chemical structures of active components}

Analysis of RP-10, using ${ }^{1} \mathrm{H}$ NMR and H-HCOSY, revealed that its major components were triglycerides (TGs) constituted of short and medium chain fatty acids. To identify the fatty acid composition, the F1-6 fraction was further saponified, methylated and analysed using GC/MS. As a result, common fatty acids, and three dicarboxylic acids, octanedioic, nonanedioic and decanedioic acids, were identified.

\subsection{Inhibition of $P G E_{2}$ production by specific fatty acids}

Of all long chain fatty acids tested, none inhibited $\mathrm{PGE}_{2}$ production in LPS-stimulated RAW264.7, but linoleic and arachidonic acids induced dose-dependent increase in the production of $\mathrm{PGE}_{2}$ (Fig. 4A). In contrast, among short and medium chain fatty acids, capric (10:0) and lauric (12:0) acids induced dose-dependent inhibition of $\mathrm{PGE}_{2}$ production in LPS-stimulated RAW264.7, and capric

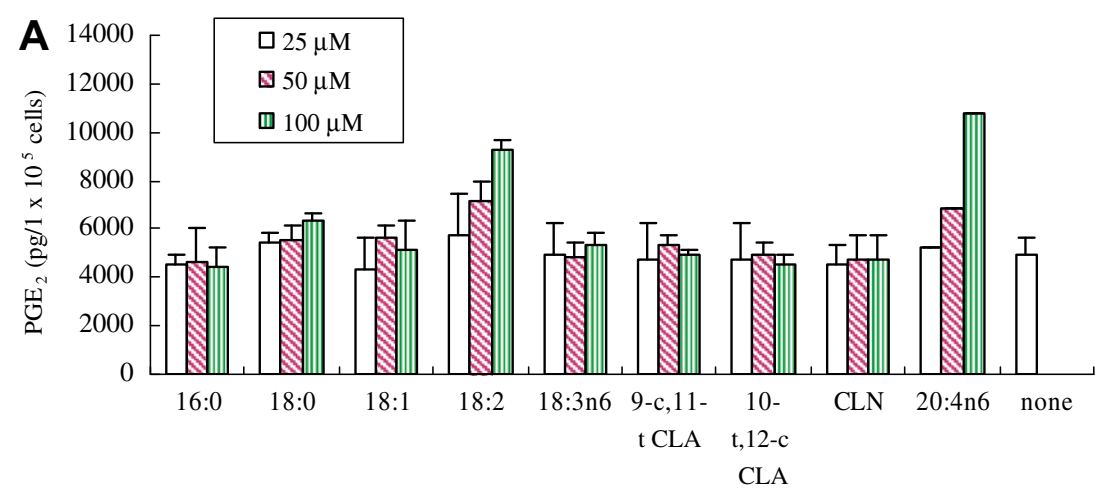

Fatty acids

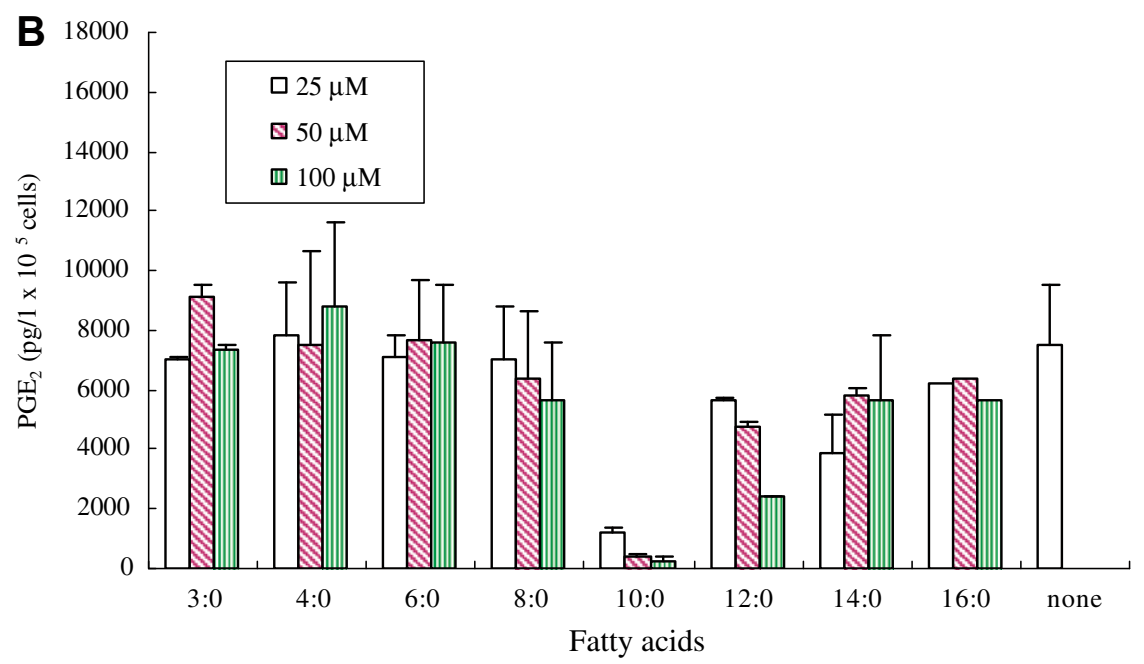

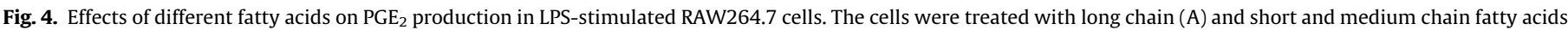

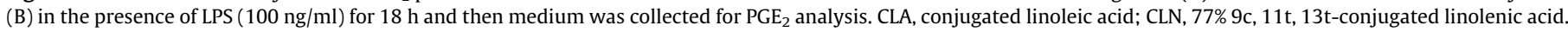
none: cells treated with LPS only. 

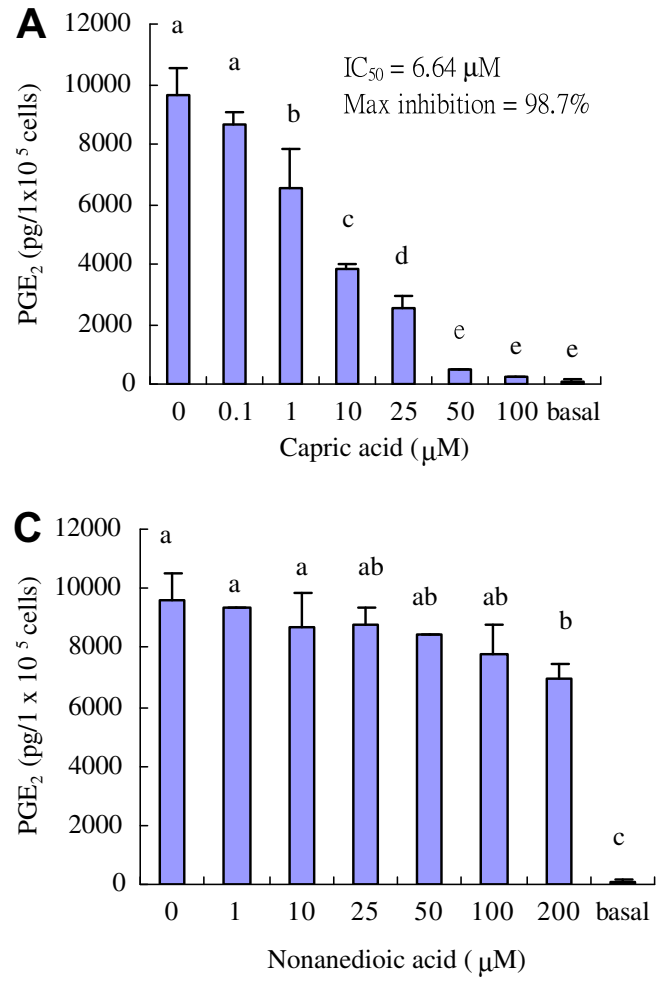
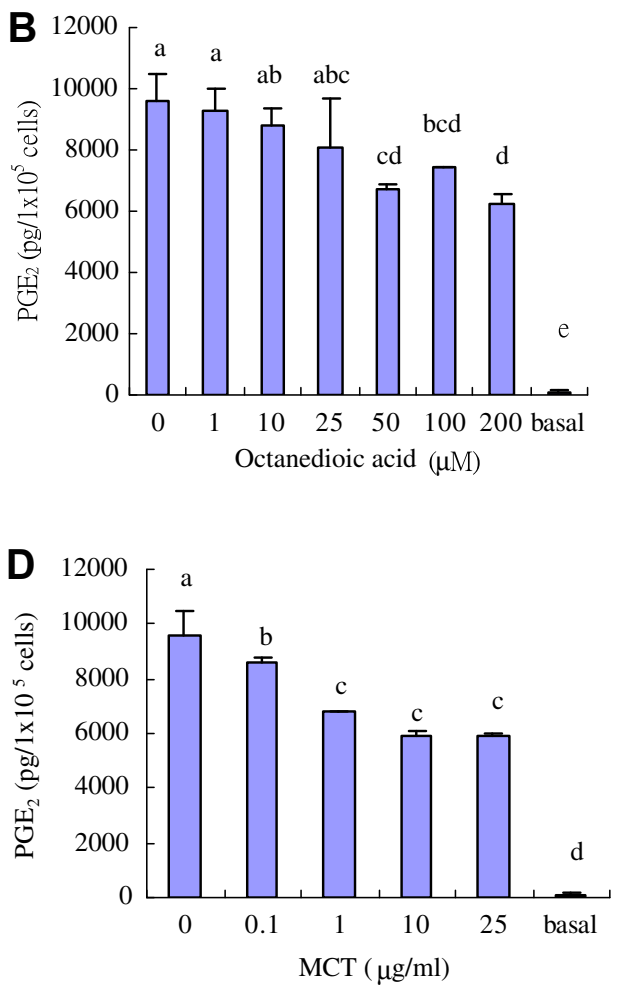

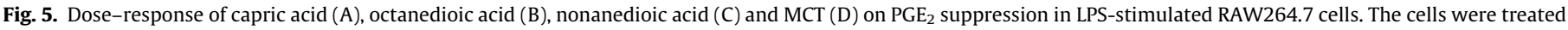

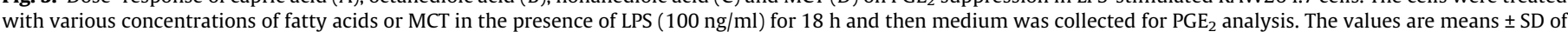

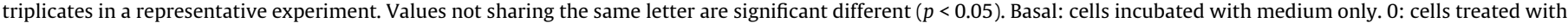
$100 \mathrm{ng} / \mathrm{ml}$ LPS only.

acid showed the highest inhibition activity (Fig. 4B). At a concentration of $50 \mu \mathrm{M}$, capric acid inhibited $\mathrm{PGE}_{2}$ production almost completely with the $\mathrm{IC}_{50}$ of $6.46 \mu \mathrm{M}(1.22 \mu \mathrm{g} / \mathrm{ml})$ (Fig. 5A). Octanedioic and nonanedioic acids had mild $\mathrm{PGE}_{2}$ inhibitory activities (Fig. 5B, C), but decanedioic acid failed to show any effect, even at concentration up to $200 \mu \mathrm{M}$ (data not shown). MCT also showed significant inhibitory activity at rather low concentration $(0.1 \mu \mathrm{g} /$ ml) (Fig. 5D).

\section{Discussion}

Surprisingly, the present study demonstrated that the components identified in the subfraction of bitter gourd EAE with the highest suppression effect of $\mathrm{PGE}_{2}$ production were TGs composed of short and medium chain fatty acids and dicarboxylic fatty acids. Due to similar polarities, it is difficult to further subfractionate these TG in terms of specific fatty acid composition and position. Noticeably, the active fractions were eluted by solvents with higher polarity than TGs with common long chain fatty acids, presumably because of their special fatty acid composition.

Recently, $\alpha$-linolenoyl-lysophosphatidylcholine (LPC) and linoleoyl-LPC, identified in butanol extract of unripened bitter gourd placenta, where the seeds are attached, were found to contribute to the suppressive effect of bitter gourd on tumor necrosis factor- $\alpha$ (TNF- $\alpha$ ) production in LPS-stimulated RAW264.7 cells (Kobori et al., 2008). Besides different parts of samples (ripened and whole vs unripened and placenta) used, our extraction procedures did not extract phospholipids or related compounds, but the effective concentration of RP-10 in this study was lower than that of purified $\alpha$-linolenoyl- or linoleoyl-LPC reported by Kobori et al. (2008). Some fractions isolated in our study, also inhibiting $\mathrm{PGE}_{2}$ production, albeit to a less extent, have not been further investi- gated. A predominant fatty acid, CLN, constituting $40 \%$ of bitter gourd EAE, is known to be a PPAR activator (Chuang et al., 2006), but did not change $\mathrm{PGE}_{2}$ production in the present study (Fig. 4B).

This study was the first to find capric acid capable of inhibiting $\mathrm{PGE}_{2}$ production in LPS-stimulated macrophages. The effective concentration of capric acid $\left(\mathrm{IC}_{50}=6.46 \mu \mathrm{M}\right.$ ) (Fig. 5A) is rather low and is likely to occur in the blood stream (Richieri \& Kleinfeld, 1995). Capric acid has also been found to be the most effective among six fatty acids in killing Gram-negative bacteria by disrupting the outer membrane (Thormar, Hilmarsson, \& Bergsson, 2006). The remaining fatty acids tested, including caprylic acid (8:0), lauric acid (12:0), mystic acid (14:0), palmitoleic acid (16:1) and oleic acid (18:1), had either no effect or only a low effect (Thormar et al., 2006). LPS is a major component of the outer membrane of Gramnegative bacteria, contributing greatly to the structural integrity of the bacteria. Capric acid might serve as a permeabiliser that induced the release of LPS from the outer membrane of Gram-negative bacteria as lactic acid did (Alakomi et al., 2000), and our observations indicated that it could simultaneously abolish the stimulation of LPS on $\mathrm{PGE}_{2}$ production of macrophages.

Only few foods contain significant amounts of capric acid, of which bovine milk fat, coconut oil and MCT are major sources. Milk fat contains $2.4 \%$ capric acid, but also contains $11 \%$ myristic acid and 33\% palmitic acid (Odongo et al., 2007). Coconut oil contains $6 \%$ capric acid, but also contains $49 \%$ lauric acid and $19 \%$ myristic acid (Laureles et al., 2002). The co-existence of atherogenic fatty acids may counteract the potential benefit of capric acid in these two oils. MCT oil, on the other hand, has a higher level of capric acid (23\%) and is devoid of atherogenic fatty acid. Our study further showed that MCT inhibited the production of $\mathrm{PGE}_{2}$ as well (Fig. 5D). Kono et al. (2003) compared the effects of MCT and corn oil on endotoxemia in rats, and found that MCT caused much less mortality and liver injury. Kupffer cells, isolated from rats given 
MCT, showed reduced LPS-stimulated TNF $\alpha$ production, and CD14 expression (Kono et al., 2003), which is a cell membrane receptor of LPS (Lu, Yeh, \& Ohashi, 2008).

The LPS-sensing machinery consists, primarily, of LPS-binding protein (LBP), CD14, and toll-like receptor 4 (TLR4), a signal-transducing integral membrane protein (Lu et al., 2008). Upon binding of LPS to cells, the sensing machinery then triggers intracellular signalling that leads to the expression of genes encoding proinflammatory cytokines or enzymes producing inflammatory molecules through the transcription factor nuclear factor $\kappa B(\mathrm{NF} \kappa \mathrm{B})$. The $\mathrm{PGE}_{2}$ production of LPS-stimulated macrophage is known to be mediated by inducing COX-2 expression through the LPS-sensing machinery pathway (Lee \& Hwang, 2006). The COX-2 expression and activity is considered the primary limiting factor for PG production in activated macrophages.

It has been demonstrated that saturated fatty acids, and especially lauric acid, which is the predominant fatty acid acylated in lipid A of LPS, could induce NFKB activation and expression of COX-2 in macrophages (Lee, Sohn, Rhee, \& Hwang, 2001). The acylation and types of acylated fatty acids of lipid A determine the biological activities of LPS (Lee \& Hwang, 2006). Conversely, unsaturated fatty acids inhibit lauric acid- or LPS-induced COX-2 expression (Lee et al., 2001). However, our results showed capric acid to be highly effective, and lauric acid (12:0) to a smaller extent, in inhibiting $\mathrm{PGE}_{2}$ production, while linoleic acid (18:2n6) and arachidonic acid (20:4n6) enhanced $\mathrm{PGE}_{2}$ production. We also found that the co-treatment with LPS and capric acid did not alter COX-2 protein expression in RAW 264.7 cells compared to treatment with LPS alone (data not shown). The discrepancy in the effect of saturated fatty acids on LPS-stimulated macrophages between our and Lee et al.'s (2003) studies might be partly explained by the differences in fatty acids tested (capric acid vs lauric acid) and experimental conditions (simultaneous treatment with fatty acids and LPS vs treatment with fatty acids prior to LPS) since lauric acid alone could have stimulated COX-2 expression (Lee et al., 2001). Besides the role of COX-2 expression, the activity of COX-2 and the availability of arachidonic acid, which is derived from the hydrolysis of phospholipids catalysed by phospholipase $\mathrm{A}_{2}$, also affect the biosynthesis of $\mathrm{PGE}_{2}$. The effects of capric acid on COX-2 activity and phospholipase $\mathrm{A}_{2}$ expression and activity need further investigation.

In conclusion, the triglycerides composed of short and medium chain saturated fatty acids and dicarboxylic acids are the active components in bitter gourd EAE contributing to the inhibition of $\mathrm{PGE}_{2}$ production, and capric acid is the most potent inhibitor among various fatty acids. Results reported here should prompt studies in other food products with similar triglyceride or fatty acid profiles. However, the potential benefits of the bioactive compounds in vegetables will need to be demonstrated in human trials.

\section{Acknowledgement}

This study was funded by a Grant, NSC 95-2317-B-003-001, from the National Science Council of the Republic of China, Taiwan.

\section{References}

Alakomi, H. L., Skyttä, E., Saarela, M., Mattila-Sandholm, T., Latva-Kala, K., \& Helander, I. M. (2000). Lactic acid permeabilizes gram-negative bacteria by disrupting the outer membrane. Applied and Environmental Microbiology, 66, 2001-2005.
Arias-Negrete, S., Keller, K., \& Chadee, K. (1995). Proinflammatory cytokines regulate cyclooxygenase-2 mRNA expression in human macrophages. Biochemical and Biophysical Research Communications, 208, 582-589.

Chuang, C. Y., Hsu, C., Chao, C. Y., Wein, Y. S., Kuo, Y. H., Huang, C., et al. (2006). Fractionation and identification of $9 \mathrm{c}, 11 \mathrm{t}, 13 \mathrm{t}$-conjugated linolenic acid as an activator of PPARalpha in bitter gourd (Momordica charantia L.). Journal of Biomedical Science, 13, 763-772.

Cipollone, F., Fazia, M., Iezzi, A., Ciabattoni, G., Pini, B., Cuccurullo, C., et al. (2004). Balance between PGD synthase and PGE synthase is a major determinant of atherosclerotic plaque instability in humans. Arteriosclerosis, Thrombosis, and Vascular Biology, 24, 1259-1265.

Denizot, F., \& Lang, R. (1986). Rapid colorimetric assay for cell growth and survival: Modifications of the tetrazolium dye procedure giving improved sensitivity and reliability. Journal of Immunological Methods, 89, 271-277.

Gómez-Hernández, A., Martín-Ventura, J. L., Sánchez-Galán, E., Vidal, C., Ortego, M. Blanco-Colio, L. M., et al. (2006). Overexpression of COX-2, Prostaglandin E synthase-1 and prostaglandin E receptors in blood mononuclear cells and plaque of patients with carotid atherosclerosis: Regulation by nuclear factorkappaB. Atherosclerosis, 187, 139-149.

Guadagni, F., Ferroni, P., Palmirotta, R., Del Monte, G., Formica, V., \& Roselli, M. (2007). Non-steroidal anti-inflammatory drugs in cancer prevention and therapy. Anticancer Research, 27, 3147-3162.

Huang, C. j., \& Wu, M. C. (2002). Differential effects of foods traditionally regarded as 'heating' and 'cooling' on prostaglandin $\mathrm{E}_{2}$ production by a macrophage cell line. Journal of Biomedical Science, 9, 596-606.

Kobori, M., Nakayama, H., Fukushima, K., Ohnishi-Kameyama, M., Ono, H. Fukushima, T., et al. (2008). Bitter gourd suppresses lipopolysaccharideinduced inflammatory responses. Journal of Agricultural and Food Chemistry. 56, 4004-4011.

Kono, H., Fujii, H., Asakawa, M., Yamamoto, M., Matsuda, M., Maki, A., et al. (2003) Protective effects of medium-chain triglycerides on the liver and gut in rats administered endotoxin. Annals of Surgery, 237, 246-255.

Laureles, L. R., Rodriguez, F. M., Reaño, C. E., Santos, G. A., Laurena, A. C., \& Mendoza, E. M. (2002). Variability in fatty acid and triacylglycerol composition of the oil of coconut (Cocos nucifera L.) hybrids and their parentals. Journal of Agriculture and Food Chemistry, 50, 1581-1586.

Lee, J. Y., Sohn, K. H., Rhee, S. H., \& Hwang, D. (2001). Saturated fatty acids, but not unsaturated fatty acids, induce the expression of cyclooxygenase- 2 mediated through toll-like receptor 4. Journal of Biological Chemistry, 276, 16683-16689.

Lee, J. Y., Plakidas, A., Lee, W. H., Heikkinen, A., Chanmugam, P., Bray, G., et al. (2003). Differential modulation of toll-like receptors by fatty acids: Preferential inhibition by $n-3$ polyunsaturated fatty acids. Journal of Lipid Research, 44 479-486.

Lee, J. Y., \& Hwang, D. H. (2006). The modulation of inflammatory gene expression by lipids: Mediation through toll-like receptors. Molecules and Cells, 21, 174-185.

Lu, Y. C., Yeh, W. C., \& Ohashi, P. S. (2008). LPS/TLR4 signal transduction pathway. Cytokine, 42, 145-451.

Mueller, H. W. (1996). Diazomethane as a highly selective fatty acid methylating reagent for use in gas chromatographic analysis. Journal of Chromatography $B$ Biomedical Application, 679, 208-209.

Odongo, N. E., Or-Rashid, M. M., Bagg, R., Vessie, G., Dick, P., Kebreab, E., et al. (2007) Long-term effects of feeding monensin on milk fatty acid composition in lactating dairy cows. Journal of Dairy Science, 90, 5126-5133.

Rhee, S. H., \& Hwang, D. (2000). Murine toll-like receptor 4 confers lipopolysaccharide responsiveness as determined by activation of NF kappa B and expression of the inducible cyclooxygenase. Journal of Biological Chemistry, 275, 34035-34040.

Richieri, G. V., \& Kleinfeld, A. M. (1995). Unbound free fatty acid levels in human serum. Journal of Lipid Research, 36, 229-240.

Robinson, D. R., McGuire, M. B., \& Levine, L. (1975). Prostaglandins in the rheumatic diseases. Annals of the New York Academy of Sciences, 256, 318-329.

Sinha, P., Clements, V. K., Fulton, A. M., \& Ostrand-Rosenberg, S. (2007). Prostaglandin $E_{2}$ promotes tumor progression by inducing myeloid-derived suppressor cells. Cancer Research, 67, 4507-4513.

Subbaramaiah, K., Yoshimatsu, K., Scherl, E., Das, K. M., Glazier, K. D., Golijanin, D., et al. (2004). Microsomal prostaglandin E synthase-1 is overexpressed in inflammatory bowel disease. Evidence for involvement of the transcription factor Egr-1. Journal of Biological Chemistry, 279, 12647-12658.

Thormar, H., Hilmarsson, H., \& Bergsson, G. (2006). Stable concentrated emulsions of the 1-monoglyceride of capric acid (monocaprin) with microbicidal activities against the food-borne bacteria Campylobacter jejuni, Salmonella spp., and Escherichia coli. Applied and Environmental Microbiology, 72, 522-526.

Williams, J. A., \& Shacter, E. (1997). Regulation of macrophage cytokine production by prostaglandin $\mathrm{E}_{2}$. Distinct roles of cyclooxygenase-1 and -2 . Journal of Biological Chemistry, 272, 25693-25699.

Wu, D., \& Meydani, S. M. (2004). Mechanism of age-associated up-regulation in macrophage $\mathrm{PGE}_{2}$ synthesis. Brain, Behavior, and Immunity, 18, 487-494. 